\title{
Simulation of an aerodynamic profile with sections of ad hoc concavity
}

\author{
Irina Pocebneva ${ }^{1,{ }^{*}}$ Yuri Deniskin ${ }^{2}$, Alexandr Yerokhin $^{2}$, Viktor Artiukh $^{3}$ and \\ Vladimir Vershinin ${ }^{4}$ \\ ${ }^{1}$ Voronezh State Technical University, Moscow Avenue, 14, Voronezh, 394026, Russia \\ ${ }^{2}$ Moscow Aviation Institute, Volokolamskoe highway, 4, Moscow, 125993, Russia \\ ${ }^{3}$ Peter the Great St. Petersburg Polytechnic University, Polytechnicheskaya 29, St. Petersburg, \\ 195251, Russia \\ ${ }^{4}$ Moscow State University of Civil Engineering, 26, Yaroslavskoye sh., Moscow, 129337, Russia
}

\begin{abstract}
The article describes the study carried out by the authors of the use of parametric geometric models to reduce the complexity of the design of parts that follow the theoretical contour of the bearing surfaces of the aircraft. Also, an algorithm that allows eliminating the existing ad hoc concavity profile is considered.
\end{abstract}

\section{Introduction}

Reducing the time of designing aviation equipment is inextricably linked with the increased productivity of the engineer in the preparation of design documentation. One of the most powerful means of reducing the complexity of design using geometric modeling systems is parametric modeling. In [1], studies of the use of parametric geometric models carried out by the authors to reduce the complexity of the design of parts emerging on the theoretical contour of the bearing surfaces of the aircraft are described.

Further studies have shown the particular importance of the quality of the surface used for the effectiveness of parametric modeling.

To simplify the design of bearing surfaces, they are decomposed into guiding and forming lines and mathematical dependencies that make up the basis of the surface and determine its shape inside the resulting cells.

The guiding lines for the bearing surfaces are aerodynamic profiles. The forming lines, as a rule, are percentage lines for which the profiles are parameter carriers.

Thus, the aerodynamic profile is the initial information when designing the wing of an aircraft, and the requirement of maintaining its shape in the design and manufacture of the wing comes to the fore as compared with the requirements of layout, manufacturability, etc.

At present, information about the contour profiles is usually represented as an ordered discrete point basis. The main means of describing point-specific curves used in the design of longitudinal and transverse carcass lines of bearing surfaces in geometric modeling

\footnotetext{
*Corresponding author: ipocebneva@vgasu.vrn.ru
} 
systems are currently spline functions belonging to the class $\mathrm{C}^{2}$, whose equations describe the behavior of a flexible rail.

When specifying the aerodynamic profile in the form of a discrete point basis, the coordinates of the points are obtained by measuring an experimental model. In this case, errors are possible due to both the accuracy of the manufacturing model and measurement accuracy. With relatively large errors in the source data, the spline interpolating the profile bypass as well as the graphs of its derivatives have pronounced oscillations. In these cases, it is necessary to smooth the bypass by deviating from some given points. The issues of smoothing discretely defined contours are considered in [2].

To solve this problem, the interpolation method developed by A. D. Tuzov with smoothing based on the use of parametric splines is very effective. A feature of the method is that it offers a clear iterative process of smoothing and proved that this process is convergent. The advantage of this method of smoothing is that in the process of smoothing, you can fix certain points.

The error of setting the $i$-th bypass point in this method is defined as half the amount of deviation of the staff at point $i$ when it is released, while fixing the rest of the contour at the same time.

\section{Problem difinition}

As studies have shown, in some cases, the use of smoothing with cubic splines does not allow eliminating the existing ad hoc (unintended) changes in the curvature sign in the spline nodes.

An example of such a circuit is shown in Fig. 1. The figure shows the upper half of the symmetric convex profile of the vertical tail of the medium-haul passenger aircraft. This profile can be divided into two sections with different nature of ad hoc changes in the sign of curvature.

Section "A" is made up of several points of the tail section, on which concavity of the circumference takes place.

Section "B" is formed by a set of points in the middle part of the bypass, on which it has oscillations.

This paper discusses the smoothing of section "A". As can be seen from Fig. 1, the curvature of the bypass on this section has a sign opposite to the required, and its graph monotonously decreases throughout the section. The calculations showed the impossibility of removing the existing concavity according to the method of A.D. Tuzov due to the very small (10-8 ... 10-6 mm) values of the error in specifying the points of the site.

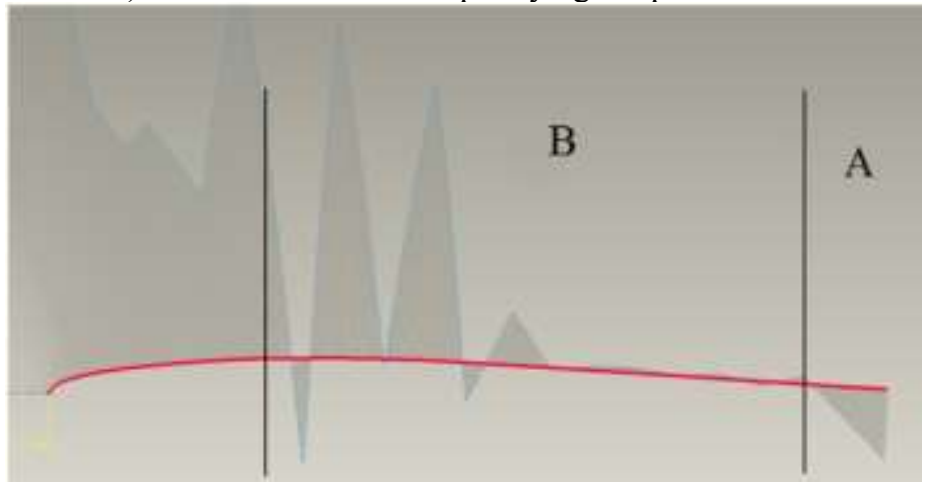

Fig. 1. Graph of the curvature of the profile sections not smoothed by the method of cubic splines 
Thus, the error $\delta \_i$ used in the method of A.D. Tuzov, geometrically interpreted as half the deviation of the elastic slats at point $i$ when it is released, is in this case not an effective enough criterion for smoothing the bypass.

The development of a smoothing technique for the type of contour under consideration requires: 1. Determining more efficient smoothness criteria than the one proposed in the method of smoothing splines; 2. Developing an algorithm to bring the coordinates of the smooth contour in accordance with the found smoothness criteria.

\section{Definition of Criteria for Smooth Contour}

To find more accurate criteria for the smoothness of the curve, the authors considered a method developed by E. V. Egorov for finding the equation of the basic integral curve for describing the lines-parameters of the aircraft surfaces described in [3]. A distinctive feature of the method is that the curve equation is searched based on the condition of its convexity.

This method allows one to find an equation with a convex curve $S(x) \in C^{2}$, passing through two points $a$ and $b$, having the second order of fixation, i.e. if at these points the values of the function are determined, its first and second derivatives (Fig. 2). The method is designed to describe the curved lines of the surface of aircraft surfaces that have a constant curvature sign on certain sections of the numerical axis; therefore, to describe them, we choose a function with a constant sign of the second derivative over the entire segment (since convex curves are considered $S^{I I}(x) \leq 0$ ).

The equation of the base integral curve is an 8th degree polynomial of the form:

$$
=a_{8} x^{8}+a_{7} x^{7}+\cdots+a_{2} x^{2}+a_{1} x+a_{0} .
$$

Due to the even degree of the polynomial used, curved lines of this type under certain boundary conditions do not always exist. Therefore, in addition to the method of determining the coefficients of equation (1), this method defines the necessary and sufficient conditions for the existence of a base integral curve used to test and change the coordinates of the original points.

In view of the special requirements imposed on the coordinates of points that serve as the initial data for constructing a basic integral curve, we assume that the boundary conditions for its existence can be used as effective smoothing criteria ensuring the constancy of the curvature sign of the smooth bypass.

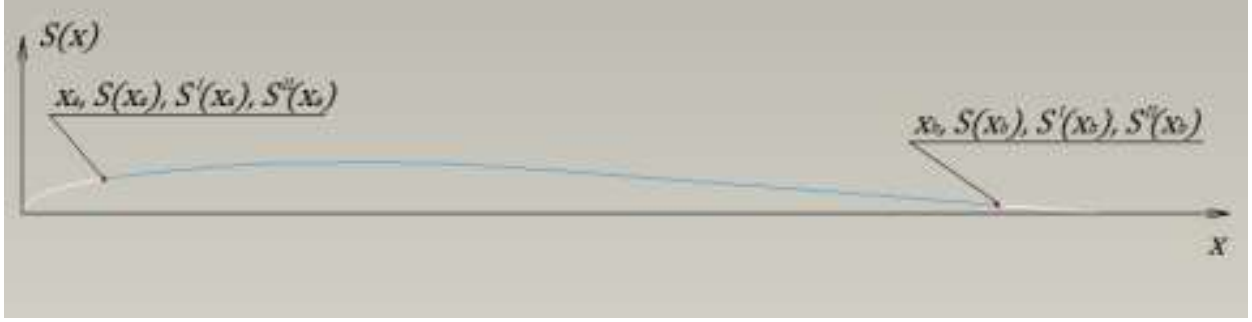

Fig. 2. Source data for constructing the base integral curve

To find the equation of the curve $\mathrm{S}(\mathrm{x})$ satisfying the given conditions, an affine transformation of the segment [x_a, $\left.\mathrm{x}_{-} \mathrm{b}\right]$ is performed. The coordinate origin is placed at the point $\mathrm{x} \_\mathrm{a}, \mathrm{S}\left(\mathrm{x} \_\mathrm{a}\right)$, while the scaling factors along the axes are equal: $\mathrm{k}_{-} \mathrm{x}=1 /\left(\mathrm{x} \_\mathrm{b}-\right.$ $\left.\mathrm{x} \_\mathrm{a}\right), \mathrm{k} \_\mathrm{y}=1$. The boundary conditions are converted to the form:

$$
x_{a}=0 ; x_{b}=1 ; S(0)=0 ; S(1)=S\left(x_{b}\right)-S\left(x_{a}\right) ;
$$




$$
\begin{array}{cl}
S^{I}(0)=S^{\prime}\left(x_{a}\right)\left(x_{b}-x_{a}\right) ; & S^{I}(1)=S^{I}\left(x_{b}\right)\left(x_{b}-x_{a}\right) ; \\
S^{I I}(0)=S^{I I}\left(x_{a}\right)\left(x_{b}-x_{a}\right)^{2} ; & S^{I I}(1)=S^{I I}\left(x_{b}\right)\left(x_{b}-x_{a}\right)^{2} .
\end{array}
$$

To find the coefficients $a_{0}, a_{1}, \ldots, a_{8}$ of the equations (1), the following system of equations is solved:

Here is the expression

$$
\begin{gathered}
S^{I I}(x)=-\left(a_{0} x^{3}+b_{0} x^{2}+c_{0} x+d_{0}\right)^{2} ; \\
\bar{B}=\int_{0}^{1} x S^{I I}(x) d x ; \\
\bar{A}=-\int_{0}^{1} S^{I I}(x) d x .
\end{gathered}
$$

$$
S^{I I}(x)=-\left(a_{0} x^{3}+b_{0} x^{2}+c_{0} x+d_{0}\right)^{2}
$$

this, according to the accepted assumption, the equation of the second derivative of the desired approximating function, from which, having $x=0$, one can find the coefficient $d_{0}=\sqrt{-S^{I I}(0)}$.

Equations

$$
\bar{B}=\int_{0}^{1} x S^{I I}(x) d x
$$

and

$$
\bar{A}=-\int_{0}^{1} S^{I I}(x) d x
$$

in which

$$
\begin{gathered}
\bar{B}=S(1)-S(0)-S^{I}(1), \\
\bar{A}=S^{I}(1)-S^{I}(0),
\end{gathered}
$$

are received subject to $S^{I I}(x) \leq 0$ of the following expressions:

$$
\begin{aligned}
& S(1)=S(0)+S^{I}(0)+\int_{0}^{1}(1-x) S^{I I}(x) d x \\
& S^{I}(1)=S^{I}(0)+\int_{0}^{1} S^{I I}(x) d x
\end{aligned}
$$

These relationships are derived in the theoretical foundations of finding the base integral curve [4] from the energy justification of its existence.

Necessary conditions for the existence of a base integral curve.

The necessary conditions for the existence of a basic integral curve are determined by the properties of polynomials of even degrees and the features of the geometric method proposed for solving the system of equations (2).

Necessary conditions for the existence of a base integral curve determined by the properties of an even degree polynomial.

For the existence of a polynomial of even degree passing through two points, it is necessary that the second derivatives of the approximated function have the same sign at both points. Taking into account the convexity condition of the approximable function, we obtain the following necessary conditions for the existence of a base integral curve [5]:

$$
\begin{aligned}
& U_{1}=S^{I I}(0) \leq 0 ; \\
& U_{2}=S^{I I}(1) \leq 0 .
\end{aligned}
$$

\section{Geometric representation of the system of equations for determining the coefficients of the base integral curve}


Consideration of equation (3), as well as equations (4) and (5) after substituting (3) into them, shows that all three equations for unknown coefficients $a_{0}, b_{0}$ and $c_{0}$ are equations of second-order surfaces (quadrics).

Equation (3) is an equation of a pair of real parallel planes. At the same time, the planes will not coincide with $S^{I I}(1) \neq 0$ and matched with $S^{I I}(1)=0$.

Equation (4) is a triaxial ellipsoid, provided that:

$$
S(1)-S(0)-S^{I}(1)>0 \text {. }
$$

Equation (5) is a triaxial ellipsoid, provided that:

$$
S^{I I}(0)>15.93975903\left(S^{I}(1)-S^{I}(0)\right)
$$

Thus, two more necessary conditions for the existence of a basic integral curve are inequalities, under which equations (4) and (5) determine the surfaces of real triaxial ellipsoids. We write these inequalities in the following form:

$$
\begin{gathered}
U_{3}=S(1)-S(0)-S^{I}(1)>0 ; \\
U_{4}=S^{I I}(0)-15.93975903\left(S^{I}(1)-S^{I}(0)\right)>0 .
\end{gathered}
$$

When conditions (10) and (11) are fulfilled, solving the system of equations (2) from a geometric point of view is finding common points belonging to the surfaces of ellipsoids and two parallel planes (Fig. 3).

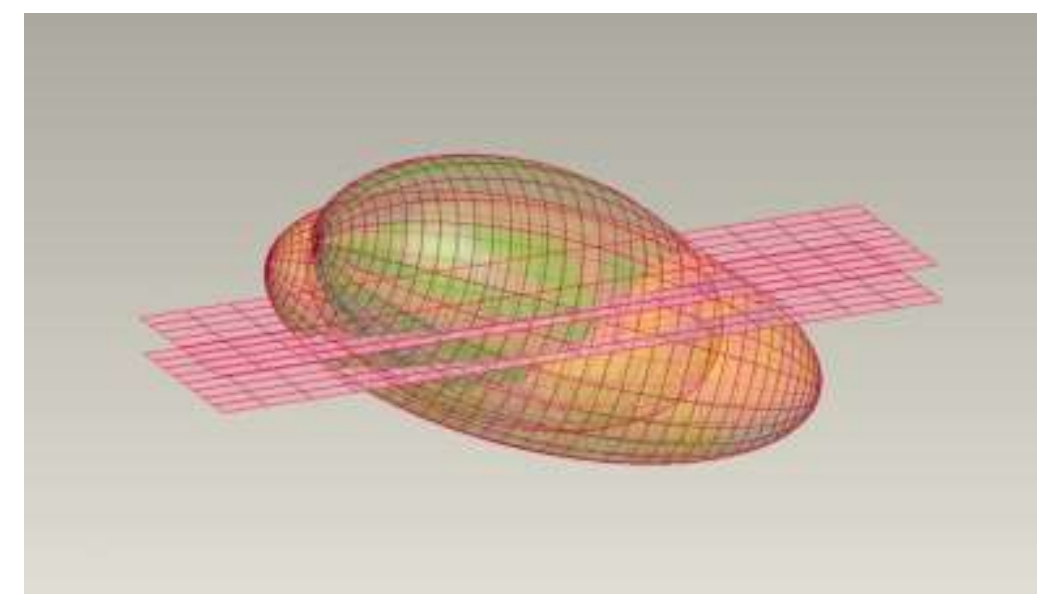

Fig. 3. Geometric solution of a system of algebraic equations

Since the plane intersects a triaxial ellipsoid along an ellipse, the solution of the system is reduced to finding the intersection points of two ellipses obtained by cutting two ellipsoids by the first plane and two other ellipses obtained by cutting the ellipsoids by the second plane.

The fulfillment of conditions (10) and (11) is necessary for the existence of real secondorder surfaces, but does not answer the question of the possibility of their intersection, i.e. obtain a valid solution to system (2).

\section{A. Sufficient conditions for the existence of a basic integral curve}

Sufficient conditions for the existence of solutions of a system of equations are determined by the conditions for the existence of real ellipses obtained when the ellipsoids are cut by parallel planes. In [6], it was shown that a sufficient condition for the existence of a basic integral curve is the simultaneous fulfillment of at least one of the two pairs of inequalities: 


$$
\begin{gathered}
T_{1}=\left\{\begin{array}{c}
-4.72 S^{I I}(1)+1.95 \sqrt{S^{I I}(0) S^{I I}(1)}+0.48 S^{I I}(0) \\
-85.03\left(S(1)-S(0)-S^{I}(1)\right)
\end{array}\right\}<0, \\
T_{2}=\left\{-2 S^{I I}(1)+\sqrt{S^{I I}(0) S^{I I}(1)}-2 S^{I I}(0)+30\left(S^{I}(1)-S^{I}(0)\right)\right\}<0 ;
\end{gathered}
$$

or

$$
\begin{gathered}
T_{3}=\left\{\begin{array}{c}
-4.72 S^{I I}(1)-1.95 \sqrt{S^{I I}(0) S^{I I}(1)}-0.48 S^{I I}(0) \\
-85.03\left(S(1)-S(0)-S^{I}(1)\right)
\end{array}\right\}<0, \\
T_{4}=\left\{-2 S^{I I}(1)-\sqrt{S^{I I}(0) S^{I I}(1)}-2 S^{I I}(0)+30\left(S^{I}(1)-S^{I}(0)\right)\right\}<0 .
\end{gathered}
$$

In the most general case, the number of solutions of the system of equations (3) - (5) is equal to eight (Fig. 4).

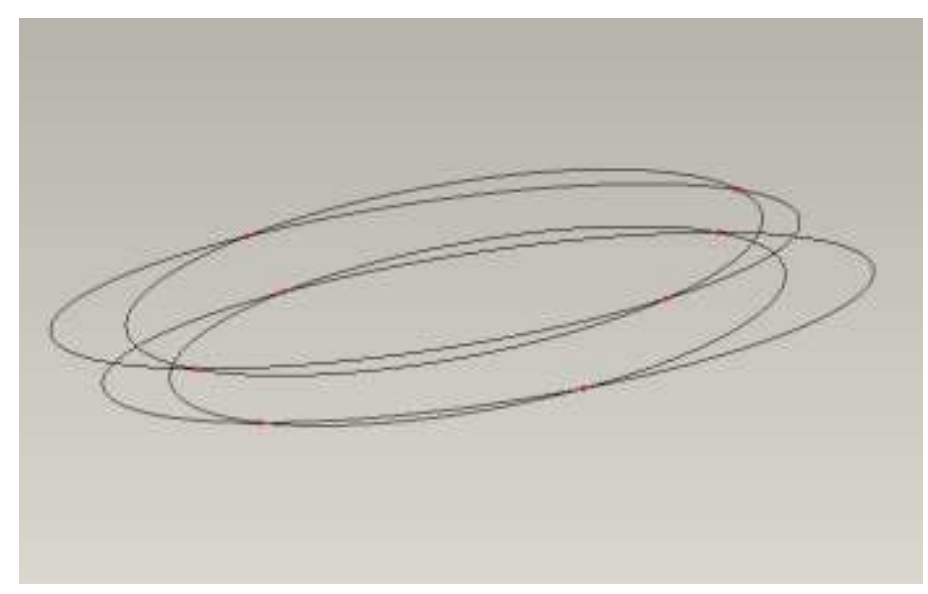

Fig. 4. Intersection points of ellipses corresponding to solutions of the system of equations

If the source data satisfies inequalities (12) and (13), then a joint solution of the equations of ellipses obtained from the cross section of ellipsoids by the first plane can give from one to four solutions determining the coefficients of equation (3).

The joint solution of the equations of ellipses obtained from the intersection of ellipsoids by a second parallel plane, provided that the initial data is satisfied with inequalities (14) and (15), gives from one to four more solutions.

Methods of solving a system of equations and finding coefficients $a_{0}, \ldots, a_{8}$, as well as the method of choosing the optimal of the obtained equations of the base integral curve, are described in detail in [7].

\section{B. Criteria for smooth contour}

The authors proposed criteria for the smoothness of a curve passing through any two points $a$ and $b$, based on the use of necessary and sufficient conditions for the existence of a basic integral curve.

To check the points for compliance with the criteria of smoothness, it requires the following:

1. Enter the approximating smoothing bypass function. $S(x)$. To assume:

$$
S\left(x_{a}\right)=y_{a}, \quad S\left(x_{b}\right)=y_{b}
$$


2. To ensure the second order of fixation points $a$ and $b$, calculate using any method the values of the first and second derivatives of the approximating function at these points:

$$
S^{I}\left(x_{a}\right), \quad S^{I}\left(x_{b}\right), \quad S^{I I}\left(x_{a}\right), \quad S^{I I}\left(x_{b}\right) .
$$

3. To carry out the valuation of the segment $\left[x_{a}, x_{b}\right]$, i.e. bring the source data to the form:

$$
\begin{gathered}
x_{a}=0 ; \quad x_{b}=1 ; S(0)=0 ; S(1)=S\left(x_{b}\right)-S\left(x_{a}\right) ; \\
S^{I}(0)=S^{I}\left(x_{a}\right)\left(x_{b}-x_{a}\right) ; S^{I}(1)=S^{I}\left(x_{b}\right)\left(x_{b}-x_{a}\right) ; \\
S^{I I}(0)=S^{I I}\left(x_{a}\right)\left(x_{b}-x_{a}\right)^{2} ; S^{I I}(1)=S^{I I}\left(x_{b}\right)\left(x_{b}-x_{a}\right)^{2} .
\end{gathered}
$$

4. Check the implementation of inequalities:

$$
\begin{gathered}
U_{1}=S^{I I}(0) \leq 0 ; \quad U_{2}=S^{I I}(1) \leq 0 \\
U_{3}=S(1)-S(0)-S^{I}(1)>0 ; \\
U_{4}=S^{I I}(0)-15.93975903\left(S^{I}(1)-S^{I}(0)\right)>0 .
\end{gathered}
$$

If all the inequalities are fulfilled, the points $a$ and $b$ satisfy the necessary conditions for the existence of a base integral curve.

We call the fulfillment of these inequalities the necessary criteria for smoothness.

5. Check the execution of pairs of inequalities:

$$
\begin{gathered}
T_{1}=\left\{-4.72 S^{I I}(1)+1.95 \sqrt{S^{I I}(0) S^{I I}(1)}+0.48 S^{I I}(0)-85.03\left(S(1)-S(0)-S^{I}(1)\right)\right\} \\
<0, \\
T_{2}=\left\{-2 S^{I I}(1)+\sqrt{S^{I I}(0) S^{I I}(1)}-2 S^{I I}(0)+30\left(S^{I}(1)-S^{I}(0)\right)\right\}<0
\end{gathered}
$$

and

$$
\begin{gathered}
T_{3}=\left\{-4.72 S^{I I}(1)-1.95 \sqrt{S^{I I}(0) S^{I I}(1)}-0.48 S^{I I}(0)-85.03\left(S(1)-S(0)-S^{I}(1)\right)\right\} \\
<0, \\
T_{4}=\left\{-2 S^{I I}(1)-\sqrt{S^{I I}(0) S^{I I}(1)}-2 S^{I I}(0)+30\left(S^{I}(1)-S^{I}(0)\right)\right\}<0 .
\end{gathered}
$$

When performing at least one of two pairs, the points $a$ and $b$ satisfy sufficient conditions for the existence of a base integral curve.

We call the fulfillment of these inequalities sufficient smoothness criteria.

Thus, the listed smoothness criteria can be used to check and refine the ordinates of the points of the smooth contour in order to ensure the constancy of the sign of the second derivative of the function approximating the bypass on a given segment of the numerical axis [8].

When applying these criteria for smoothing the contours depending on the convexity or concavity of the contour section under consideration, each point must be assigned a parameter $\mathrm{p}$, which can take the following values:

$p=-1$, if in the vicinity of this point the curve is concave;

$p=0$, if the second derivative is 0 at the point;

$p=1$, if the curve is convex in a neighborhood of a given point.

\section{Development of smoothing algorithm}

Let's consider the smoothing section "A" (Fig. 5). 


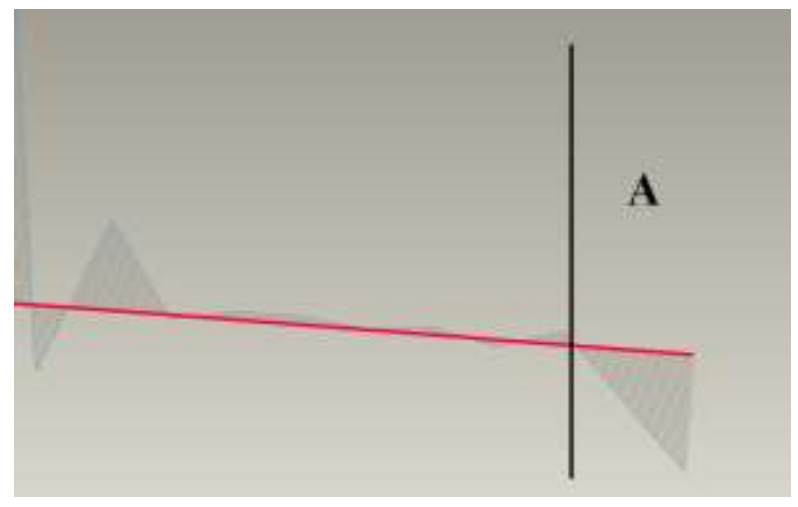

Fig. 5. Graph of curvature of section "A" of the smoothing contour

Introduce the indexing of the points of smoothing bypass:

$$
\left\{x_{i}, y_{i}\right\}, \quad i=0,1,2, \ldots, n \text {. }
$$

Since to check whether the criteria for the smoothness of the bypass are met, points $a$ and $b$ should have a second order of fixation, it is required to determine the values of the first and second derivatives of the approximating function at these points.

To calculate the first and second derivatives at the points of the studied bypass, the authors used a simplified method for modeling convex curves, proposed in [9], to calculate the initial data when finding the equation of the basic integral curve.

\section{Application of a simplified method for modeling convex curves to determine the first and second derivatives of the approximating function}

The method consists in taking the values of the first and second derivatives of the approximating function $S(x)$ at the $i$-th point equal, respectively, to the first and second derivatives of the square parabola of the form

drawn through the points $i-1, i, i+1$.

$$
Y(x)=k_{2} x^{2}+k_{1} x+k_{0}
$$

This is possible under the assumption that in any sufficiently close neighborhood of the $i$-th point, the searched curve $S(x)$ and the parabola $Y(x)$ satisfy the conditions:

$$
S\left(x_{i}\right)=Y\left(x_{i}\right), \quad\left|S^{I}\left(x_{i}\right)-Y^{I}\left(x_{i}\right)\right|<\varepsilon_{1}, \quad\left|S^{I I}\left(x_{i}\right)-Y^{I I}\left(x_{i}\right)\right|<\varepsilon_{2},
$$

where $\varepsilon_{1}, \varepsilon_{2}$ - preassigned infinitesimal numbers, and $\varepsilon_{1} \varepsilon_{2} \rightarrow 0$ at $x-x_{i \pm 0} \rightarrow 0$.

The assumption about the validity of the assumptions made for curves describing the contours in aircraft manufacturing is justified in [10] with the help of experimental data.

Taking into account the introduced assumptions, the first and second derivatives of the approximating function are calculated in the following sequence. First, by coordinates of points $i-1, i, i+1$, the coefficients $k_{2}$ and $k_{1}$ of equation (16) are found (the coefficient $k_{0}$ is not required to calculate the derivatives):

$$
\begin{aligned}
& k_{2}=\frac{y_{i+1}-\frac{x_{i+1}\left(y_{i}-y_{i-1}\right)+x_{i} y_{i-1}-x_{i-1} y_{i}}{x_{i}-x_{i-1}}}{x_{i+1}\left(x_{i+1}-x_{i-1}-x_{i}\right)+x_{i-1} x_{i}}, \\
& k_{1}=\frac{y_{i}-y_{i-1}}{x_{i}-x_{i-1}}-k_{2}\left(x_{i-1}+x_{i}\right) .
\end{aligned}
$$

After that, the values of the first and second derivatives of the approximating function are determined at the point with coordinates $\left(x_{i}, y_{i}\right)$ by the formulas of the corresponding derivatives of the parabola (16):

$$
S^{I}\left(x_{i}\right)=Y^{I}\left(x_{i}\right)=2 k_{2} x_{i}+k_{1}
$$




$$
S^{I I}\left(x_{i}\right)=Y^{I I}\left(x_{i}\right)=2 k_{2} .
$$

The results of the calculation of the first and second derivatives at the points of section "A" and several points adjacent to it are given in Table 1.

Table 1. The results of the calculation of the first and second derivatives at the points of section "A" and several adjacent points

\begin{tabular}{|c|c|c|c|c|}
\hline$i$ & $X_{i}$ & $Y_{i}$ & $S^{I}$ & $S^{I I}$ \\
\hline 22 & 1534 & 63.12 & -0.075338983 & $-4.30911 \times 10^{-6}$ \\
\hline 23 & 1652 & 54.2 & -0.075889831 & $-5.02729 \times 10^{-6}$ \\
\hline 24 & 1770 & 45.21 & -0.07631 & $-2.2 \times 10^{-6}$ \\
\hline 25 & 1888 & 36.19 & -0.07661 & $-2.9 \times 10^{-6}$ \\
\hline 26 & 2006 & 27.13 & -0.07674 & $7.18 \times 10^{-7}$ \\
\hline 27 & 2124 & 18.08 & -0.07665 & $7.18 \times 10^{-7}$ \\
\hline 28 & 2242 & 9.04 & -0.07449 & $3.59 \times 10^{-5}$ \\
\hline
\end{tabular}

\section{Smoothing procedure}

The procedure for smoothing section "A" developed by the authors consists in checking the fulfillment of the smoothness criteria for the bypass on the segment $\left[x_{a}, x_{b}\right]$ and changing the value of $y_{b}$ when they fail. To ensure that the condition $U_{1} \leq 0$ is met, a point with index $m$ is selected as point $a$, which lies in the forward part of the bypass (not belonging to section "B"), in which the curve describing the bypass is rather strongly convex, i.e. the value of the second derivative of the approximating function is substantially less than zero:

$$
a=\left\{x_{m}, y_{m}\right\}, \quad 0<m<n, \quad S^{I I}\left(x_{m}\right) \ll 0 .
$$

As the point $b$, the points of the smoothed area are successively substituted. In this example, these will be points $n-1, n-2, \ldots$, with the exception of $n$.

At points a and $b$, according to formulas (19) and (20), the first and second derivatives of the approximating function are calculated, and the fulfillment of the smoothness criteria for the bypass is checked. If the criteria are not met, the $y_{b}$ is adjusted to the value at which they will be executed.

Changing the values of the ordinates of each of the points has an effect on the values of the second derivatives in the neighboring points; therefore, you should consider not only the points belonging to the smoothing section as point $b$, but all the points of the bypass between $\alpha$ (point with index $m$ ) and $n$ - the last point bypass Thus, the sequence of points selected as point $b$ will have the following form: $b_{j}=\left\{x_{i}, y_{i}\right\} ; i=n-1, n-2, \ldots, m+$ $1 ; j=1,2, \ldots, n-(m+1)$.

Correction of the ordinate point $b_{j+1}$ can change the value of the second derivative at $b_{j}$ in the way that the criteria for smoothness in $b_{j}$ will stop fulfilling. Then you need to rechange the ordinate point $b_{j}$.

Therefore, the developed process of correction of ordinate values will be iterative, and the sequence of points $b_{j}$ would look like this:

where $l$ - iteration number.

$$
b_{j}^{l}, \quad l=1,2, \ldots,
$$

In accordance with the definitions of the necessary and sufficient criteria for smoothness, it is possible to determine the necessary and sufficient conditions for completing the iterations. The necessary condition for completing the iterations will be the fulfillment of the necessary smoothness criteria without correction of the ordinates at all points. $b_{j}^{l}$ :

$$
\begin{gathered}
U_{2}\left(b_{j}^{l}\right) \leq 0, \quad U_{3}\left(a, b_{j}^{l}\right)>0, \quad U_{4}\left(a, b_{j}^{l}\right)>0, \\
j=1,2, \ldots, n-(m+1) .
\end{gathered}
$$


Accordingly, a sufficient condition for completing the iterations will be the same fulfillment of sufficient smoothness criteria at all points. $b_{j}^{l}$ :

$$
\begin{gathered}
T_{1}\left(a, b_{j}^{l}\right)<0, \quad T_{2}\left(a, b_{j}^{l}\right)<0, \\
T_{3}\left(a, b_{j}^{l}\right)<0, \quad T_{4}\left(a, b_{j}^{l}\right)<0, \\
j=1,2, \ldots, n-(m+1) .
\end{gathered}
$$

The smoothing algorithm for the contours that have areas with an extended unregulated concavity and without restrictions on the deviation from the original coordinates is described in [10]. The results of the performed smoothing showed a deviation of the ordinate of one of the points from the initial value by a sufficiently large value, $\sim 5 \%$. Large deviations from the original coordinates of the profile points may adversely affect the aerodynamic properties of the smooth contour. In engineering practice, the tolerance is considered to be within $3 \%$ of the coordinate value of the original profile.

Thus, in order to avoid possible deterioration of the aerodynamic properties of the smoothing contour, the verification of the condition of keeping the specified deviation from the original coordinates is included in the smoothing procedure. For a deviation of $3 \%$, this condition is:

$$
\left|\frac{y_{i}^{k}-y_{i}^{(0)}}{y_{i}^{(0)}}\right| \leq 0.03
$$

where $y_{i}^{(0)}$ - original ordinate value of the $i$-th point of smoothing contour, $y_{i}^{k}$ - ordinate value of the $i$-th point on the $k$-th iteration of the smoothing procedure.

If criteria of smoothness are not met on the segment $\left[a, b_{j}^{l}\right]$, achieving the negativity of the second derivative of the curve describing the bypass at the point $b_{j}^{l}=\left\{x_{i}, y_{i}\right\}$ is possible in two ways:

1. By increasing the $y_{i}$ by step size $t$;

2. By decreasing the ordinate of the point through which any of the branches of the square parabola passes through the points $i-1, i, i+1$, eg $y_{i-1}$, by step size $t$ (Fig. 6).

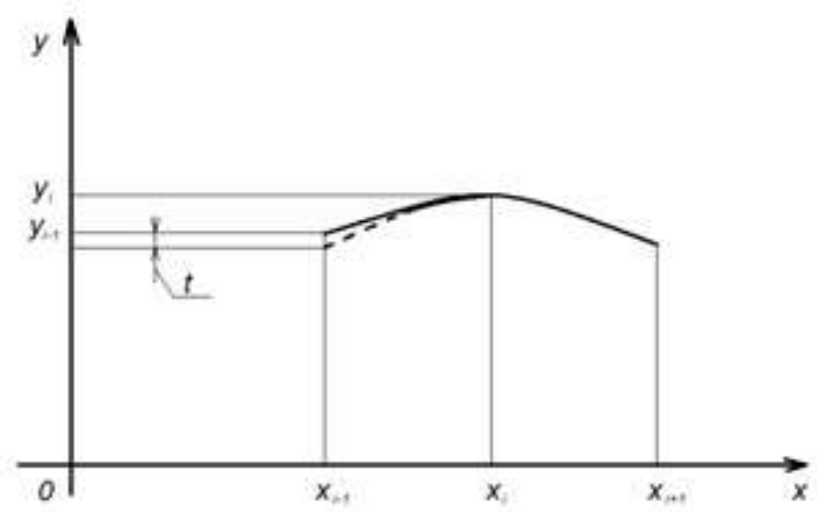

Fig. 6. Decrease of the point ordinate by step size $t$

As long as condition (21) is fulfilled, if the smoothness criteria are not met, the ordinate of the point $b_{j}^{l}$ is increased:

$$
y_{i}^{k+1}=y_{i}^{k}+t
$$

If this condition is no longer satisfied, the ordinate of the point $b_{j}^{l}$ is returned to the previous value:

$$
y_{i}^{k+1}=y_{i}^{k}-t
$$


and the smoothing algorithm changes: if the smoothness criteria are not met, the ordinate $y_{i-1}$ is decreased:

to the value at which they will be executed.

$$
y_{i-1}^{k+1}=y_{i-1}^{k}-t
$$

Thus, to smooth the section "A" of the bypass under consideration with the specified deviation from the original profile, the authors developed an algorithm for smoothing the bypass section with a long unregulated concavity with a given restriction of deviation from the original coordinates.

\section{A. Algorithm for smoothing the bypass section with an extended unregulated concavity with a given restriction of deviations from the original coordinates}

1. As a point $\alpha$, select any node with the index $m$, lying in the forward part of the bypass on the section that does not require smoothing, such as: $a=\left\{x_{m}, y_{m}\right\}, 0<m<n$, $S^{I I}\left(x_{m}\right) \ll 0$.

Assume:

$$
x_{a}=x_{m}, \quad S\left(x_{a}\right)=y_{m} .
$$

2. By coordinates of the points $m-1, m, m+1$, calculate coefficients $k_{2_{m}}$ and $k_{1_{m}}$ of the parabola passing through them:

$$
\begin{gathered}
k_{2_{m}}=\frac{y_{m+1}-\frac{x_{m+1}\left(y_{m}-y_{m-1}\right)+x_{m} y_{m-1}-x_{m-1} y_{m}}{x_{m}-x_{m-1}}}{x_{m+1}\left(x_{m+1}-x_{m-1}-x_{m}\right)+x_{m-1} x_{m}}, \\
k_{1_{m}}=\frac{y_{m}-y_{m-1}}{x_{m}-x_{m-1}}-k_{2_{m}}\left(x_{m-1}+x_{m}\right) .
\end{gathered}
$$

3. Determine the values of the first and second derivatives of the approximating function at the point $m$ :

$$
\begin{gathered}
S^{I}\left(x_{a}\right)=Y^{I}\left(x_{m}\right)=2 k_{2 m} x_{i}+k_{1_{m}}, \\
S^{I I}\left(x_{a}\right)=Y^{I I}\left(x_{m}\right)=2 k_{2_{m}} .
\end{gathered}
$$

4. Set $t$ - step of correction of the ordinate value of a point $b$.

5. Assume $l=1$.

6 . Assume $j=1$.

7.Define point $b_{j}^{l}$ :

Assume:

$$
b_{j}^{l}=\left\{x_{i}, y_{i}\right\} ; i=n-1, n-2, \ldots, m+1 ; j=1,2, \ldots, n-(m+1) .
$$

$$
x_{b_{j}^{l}}=x_{i}, \quad S\left(x_{b_{j}^{l}}\right)=y_{i}
$$

8. By coordinates of the points $i-1, i, i+1$, calculate coefficient $k_{2}$ and $k_{1}$ of the parabola passing through them:

$$
\begin{gathered}
k_{2_{i}}=\frac{y_{i+1}-\frac{x_{i+1}\left(y_{i}-y_{i-1}\right)+x_{i} y_{i-1}-x_{i-1} y_{i}}{x_{i}-x_{i-1}}}{x_{i+1}\left(x_{i+1}-x_{i-1}-x_{i}\right)+x_{i-1} x_{i}}, \\
k_{1_{i}}=\frac{y_{i}-y_{i-1}}{x_{i}-x_{i-1}}-k_{2_{i}}\left(x_{i-1}+x_{i}\right) .
\end{gathered}
$$

9. Determine the values of the first and second derivatives of the approximating function at the point $i$ :

$$
S^{I}\left(x_{b_{j}^{l}}\right)=Y^{I}\left(x_{i}\right)=2 k_{2 i} x_{i}+k_{1_{i}}
$$




$$
S^{I I}\left(x_{b_{j}^{l}}\right)=Y^{I I}\left(x_{i}\right)=2 k_{2_{i}} .
$$

10. Perform segment normalization $\left[a, b_{j}^{l}\right]$. Convert the boundary conditions to the form:

$$
\begin{gathered}
x_{a}=0 ; \quad x_{b_{j}^{l}}=1 ; S(0)=0 ; S(1)=S\left(x_{b_{j}^{l}}\right)-S\left(x_{a}\right) ; \\
S^{I}(0)=S^{\prime}\left(x_{a}\right)\left(x_{b_{j}^{l}}-x_{a}\right) ; S^{I}(1)=S^{I}\left(x_{b_{j}^{l}}\right)\left(x_{b_{j}^{l}}-x_{a}\right) ; \\
S^{I I}(0)=S^{I I}\left(x_{a}\right)\left(x_{b_{j}^{l}}-x_{a}\right)^{2} ; S^{I I}(1)=S^{I I}\left(x_{b_{j}^{l}}\right)\left(x_{b_{j}^{l}}-x_{a}\right)^{2} .
\end{gathered}
$$

11. Check the fulfillment of the required criteria for smoothness:

$$
\begin{gathered}
U_{1}=S^{I I}(0) \leq 0 ; \quad U_{2}=S^{I I}(1) \leq 0 ; \\
U_{3}=S(1)-S(0)-S^{I}(1)>0 ; \\
U_{4}=S^{I I}(0)-15.93975903\left(S^{I}(1)-S^{I}(0)\right)>0 .
\end{gathered}
$$

11.1 When performing all inequalities, go to step 12 .

11.2 If any of the inequalities fails, assume that

11.2.1 Check the condition

$$
y_{i}^{k+1}=y_{i}^{k}+t
$$

$$
\left|\frac{y_{i}^{k+1}-y_{i}^{0}}{y_{i}^{0}}\right| \leq 0.03 .
$$

11.2.1.1 When the condition is met, go to step 7.

11.2.1.2 If the condition is not met, assume that

and go to step 18 .

$$
y_{i}^{k+1}=y_{i}^{k}-t
$$

12 Verify that sufficient smoothness criteria are met:

$$
\begin{gathered}
T_{1}=\left\{-4,72 S^{I I}(1)+1,95 \sqrt{S^{I I}(0) S^{I I}(1)}+0,48 S^{I I}(0)-85,03\left(S(1)-S(0)-S^{I}(1)\right)\right\} \\
<0, \\
T_{2}=\left\{-2 S^{I I}(1)+\sqrt{S^{I I}(0) S^{I I}(1)}-2 S^{I I}(0)+30\left(S^{I}(1)-S^{I}(0)\right)\right\}<0 ; \\
T_{3}=\left\{-4,72 S^{I I}(1)-1,95 \sqrt{S^{I I}(0) S^{I I}(1)}-0,48 S^{I I}(0)-85,03\left(S(1)-S(0)-S^{I}(1)\right)\right\} \\
<0, \\
T_{4}=\left\{-2 S^{I I}(1)-\sqrt{S^{I I}(0) S^{I I}(1)}-2 S^{I I}(0)+30\left(S^{I}(1)-S^{I}(0)\right)\right\}<0 .
\end{gathered}
$$

12.1 If inequalities $T_{1}<0$ and $T_{2}<0$, or $T_{3}<0$ and $T_{4}<0$ are met simultaneously, go to step 13 .

12.2 If inequalities $T_{1}<0$ and $T_{2}<0$, or $T_{3}<0$ and $T_{4}<0$ are not met simultaneously, assume that

12.2.1 Check the condition

$$
y_{i}^{k+1}=y_{i}^{k}+t
$$

$$
\left|\frac{y_{i}^{k+1}-y_{i}^{0}}{y_{i}^{0}}\right| \leq 0.03 .
$$

12.2.1.1 If the condition is met, go to step 7.

12.2.1.2 If the condition is not met, assume that

and go to step 18 .

$$
y_{i}^{k+1}=y_{i}^{k}-t
$$

13 Assume that $j=j+1$.

14 Check the condition

$$
j \leq n-(m+1) .
$$


14.1 If the condition is met, go to step 7.

14.2 If the condition is not met, go to step 15 .

15 Check the necessary condition for completing the iterations - meeting the necessary smoothness criteria without correction of the ordinates at all points $b_{j}^{l}$ :

$U_{2}\left(b_{j}^{l}\right) \leq 0, \quad U_{3}\left(a, b_{j}^{l}\right)>0, \quad U_{4}\left(a, b_{j}^{l}\right)>0, \quad j=1,2, \ldots, n-(m+1)$.

15.1 If the condition is met, go to step 16 .

15.2 If the condition is not met, assume that

$$
l=l+1
$$

and go to step 6 .

16 Check the necessary condition for completing the iterations - meeting the sufficient smoothness criteria without correction of the ordinates at all points $b_{j}^{l}$ :

$$
\begin{gathered}
T_{1}\left(a, b_{j}^{l}\right)<0, \quad T_{2}\left(a, b_{j}^{l}\right)<0, \quad T_{3}\left(a, b_{j}^{l}\right)<0, \quad T_{4}\left(a, b_{j}^{l}\right)<0, \\
j=1,2, \ldots, n-(m+1) .
\end{gathered}
$$

16.1 If inequalities $T_{1}\left(a, b_{j}^{l}\right)<0$ and $T_{2}\left(a, b_{j}^{l}\right)<0$, or $T_{3}\left(a, b_{j}^{l}\right)<0$ and $T_{4}\left(a, b_{j}^{l}\right)<0$ are not met simultaneously for any value of $j$, assume that

and go to step 6 .

$$
l=l+1
$$

16.2 If inequalities $T_{1}\left(a, b_{j}^{l}\right)<0$ and $T_{2}\left(a, b_{j}^{l}\right)<0$, or $T_{3}\left(a, b_{j}^{l}\right)<0$ and $T_{4}\left(a, b_{j}^{l}\right)<0$ are met simultaneously for all values of $j$, the calculation is over.

$17 \quad$ Assume that $j=1$.

18 Determine the point $b_{j}^{l}$ :

Assume that:

$$
b_{j}^{l}=\left\{x_{i}, y_{i}\right\} ; i=n-1, n-2, \ldots, m+1 ; j=1,2, \ldots, n-(m+1) .
$$

$$
x_{b_{j}^{l}}=x_{i}, \quad S\left(x_{b_{j}^{l}}\right)=y_{i} .
$$

19 By coordinates of points $i-1, i, i+1$, calculate coefficients $k_{2}$ and $k_{1}$ of the parabola passing through them:

$$
\begin{gathered}
k_{2_{i}}=\frac{y_{i+1}-\frac{x_{i+1}\left(y_{i}-y_{i-1}\right)+x_{i} y_{i-1}-x_{i-1} y_{i}}{x_{i}-x_{i-1}}}{x_{i+1}\left(x_{i+1}-x_{i-1}-x_{i}\right)+x_{i-1} x_{i}}, \\
k_{1_{i}}=\frac{y_{i}-y_{i-1}}{x_{i}-x_{i-1}}-k_{2_{i}}\left(x_{i-1}+x_{i}\right) .
\end{gathered}
$$

20 Determine the values of the first and second derivatives of the approximating function at the point $i$ :

$$
\begin{gathered}
S^{I}\left(x_{b_{j}^{l}}\right)=Y^{I}\left(x_{i}\right)=2 k_{2_{i}} x_{i}+k_{1_{i}}, \\
S^{I I}\left(x_{b_{j}^{l}}\right)=Y^{I I}\left(x_{i}\right)=2 k_{2_{i}} .
\end{gathered}
$$

21 Perform rationing of the segment $\left[a, b_{j}^{l}\right]$. Convert boundary conditions to the following form:

$$
\begin{gathered}
x_{a}=0 ; x_{b_{j}^{l}}=1 ; S(0)=0 ; S(1)=S\left(x_{b_{j}^{l}}\right)-S\left(x_{a}\right) ; \\
S^{I}(0)=S^{\prime}\left(x_{a}\right)\left(x_{b_{j}^{l}}-x_{a}\right) ; S^{I}(1)=S^{I}\left(x_{b_{j}^{l}}\right)\left(x_{b_{j}^{l}}-x_{a}\right) ; \\
S^{I I}(0)=S^{I I}\left(x_{a}\right)\left(x_{b_{j}^{l}}-x_{a}\right)^{2} ; S^{I I}(1)=S^{I I}\left(x_{b_{j}^{l}}\right)\left(x_{b_{j}^{l}}-x_{a}\right)^{2} .
\end{gathered}
$$

22 Verify that the required smoothness criteria are met: 


$$
\begin{gathered}
U_{1}=S^{I I}(0) \leq 0 ; \quad U_{2}=S^{I I}(1) \leq 0 ; \\
U_{3}=S(1)-S(0)-S^{I}(1)>0 ; \\
U_{4}=S^{I I}(0)-15,93975903\left(S^{I}(1)-S^{I}(0)\right)>0 .
\end{gathered}
$$

22.1 If all inequalities are met, go to step 23 .

22.2 If any of inequalities are not met, assume that

and go to step 18 .

$$
y_{i-1}^{k+1}=y_{i-1}^{k}-t
$$

23 Verify that sufficient smoothness criteria are met:

$$
\begin{aligned}
& T_{1}=\left\{-4.72 S^{I I}(1)+1.95 \sqrt{S^{I I}(0) S^{I I}(1)}+0.48 S^{I I}(0)-85.03\left(S(1)-S(0)-S^{I}(1)\right)\right\} \\
& <0 \text {, } \\
& T_{2}=\left\{-2 S^{I I}(1)+\sqrt{S^{I I}(0) S^{I I}(1)}-2 S^{I I}(0)+30\left(S^{I}(1)-S^{I}(0)\right)\right\}<0 ; \\
& T_{3}=\left\{-4.72 S^{I I}(1)-1.95 \sqrt{S^{I I}(0) S^{I I}(1)}-0.48 S^{I I}(0)-85.03\left(S(1)-S(0)-S^{I}(1)\right)\right\} \\
& <0 \text {, } \\
& T_{4}=\left\{-2 S^{I I}(1)-\sqrt{S^{I I}(0) S^{I I}(1)}-2 S^{I I}(0)+30\left(S^{I}(1)-S^{I}(0)\right)\right\}<0 . \\
& T_{4}=\left\{-2 S^{I I}(1)-\sqrt{S^{I I}(0) S^{I I}(1)}-2 S^{I I}(0)+30\left(S^{I}(1)-S^{I}(0)\right)\right\}<0 .
\end{aligned}
$$

23.1 If inequalities $T_{1}<0$ and $T_{2}<0$, or $T_{3}<0$ and $T_{4}<0$ are met simultaneously, go to step 25 .

23.2 If inequalities $T_{1}<0$ and $T_{2}<0$, or $T_{3}<0$ and $T_{4}<0$ are not met simultaneously, assume that

and go to step 18 .

$$
y_{i}^{k+1}=y_{i}^{k}-t
$$

24 Assume that $j=j+1$.

25 Check the condition

25.1 If the condition is met, go to step 18 .

$$
j \leq n-(m+1)
$$

25.2 If the condition is not met, go to step 26.

26 Check the necessary condition for completing the iterations - meeting the necessary smoothness criteria without correction of the ordinates at all points $b_{j}^{l}$ :

$$
U_{2}\left(b_{j}^{l}\right) \leq 0, \quad U_{3}\left(a, b_{j}^{l}\right)>0, \quad U_{4}\left(a, b_{j}^{l}\right)>0, \quad j=1,2, \ldots, n-(m+1) .
$$

26.1 If the condition is met, go to step 27.

26.2 If the condition is not met, assume that

and go to step 17.

$$
l=l+1
$$

27. Check that the condition for completing the iterations is sufficient - satisfying sufficient smoothness criteria without correcting the ordinates at all points $b_{j}^{l}$ :

$$
\begin{gathered}
T_{1}\left(a, b_{j}^{l}\right)<0, \quad T_{2}\left(a, b_{j}^{l}\right)<0, \quad T_{3}\left(a, b_{j}^{l}\right)<0, \quad T_{4}\left(a, b_{j}^{l}\right)<0, \\
j=1,2, \ldots, n-(m+1) .
\end{gathered}
$$

27.1 If inequalities $T_{1}\left(a, b_{j}^{l}\right)<0$ and $T_{2}\left(a, b_{j}^{l}\right)<0$, or $T_{3}\left(a, b_{j}^{l}\right)<0$ and $T_{4}\left(a, b_{j}^{l}\right)<0$ are not met simultaneously for any value of $j$, assume that

and go to step 17.

$$
l=l+1
$$

27.2 If inequalities $T_{1}\left(a, b_{j}^{l}\right)<0$ and $T_{2}\left(a, b_{j}^{l}\right)<0$, or $T_{3}\left(a, b_{j}^{l}\right)<0$ and $T_{4}\left(a, b_{j}^{l}\right)<$ 0 are met simultaneously for all values of $j$, the calculation is over. 


\section{B. Smoothing section "A" according to the developed algorithm}

Table 2 shows the coordinates of the circumference points smoothed by the developed algorithm, as well as the values of the first and second derivatives of the approximating function at these points. Fig. 7 shows the outline and a graph of its curvature obtained by means of a geometric modeling system. The deviations of the coordinates of the smoothed contour from the source are given in Table 3.

Table 2. Coordinates of the points

\begin{tabular}{|c|c|c|c|c|}
\hline$i$ & $X_{i}$ & $Y_{i}$ & $S^{I}$ & $S^{I I}$ \\
\hline 11 & 295 & 85.59 & & \\
\hline 12 & 354 & 91.14 & 0.086497 & -0.0002566 \\
\hline 13 & 472 & 99.56 & 0.056737 & -0.0002478 \\
\hline 14 & 590 & 104.53 & 0.023898 & -0.0003088 \\
\hline 15 & 708 & 105.2 & 0.002331 & $-5.674 \times 10^{-5}$ \\
\hline 16 & 826 & 105.08 & -0.01479 & -0.0002334 \\
\hline 17 & 944 & 101.71 & -0.03716 & -0.0001458 \\
\hline 18 & 1062 & 96.31 & -0.05936 & -0.0002305 \\
\hline 19 & 1180 & 87.7 & -0.07305 & $-1.436 \times 10^{-6}$ \\
\hline 20 & 1298 & 79.07 & -0.07322 & $-1.436 \times 10^{-6}$ \\
\hline 21 & 1416 & 70.42 & -0.07339 & $-1.436 \times 10^{-6}$ \\
\hline 22 & 1534 & 61.75 & -0.07356 & $-1.436 \times 10^{-6}$ \\
\hline 23 & 1652 & 53.06 & -0.07373 & $-1.436 \times 10^{-6}$ \\
\hline 24 & 1770 & 44.35 & -0.0739 & $-1.436 \times 10^{-6}$ \\
\hline 25 & 1888 & 35.62 & -0.07407 & $-1.436 \times 10^{-6}$ \\
\hline 26 & 2006 & 26.87 & -0.07424 & $-1.436 \times 10^{-6}$ \\
\hline 27 & 2124 & 18.1 & -0.07441 & $-1.436 \times 10^{-6}$ \\
\hline 28 & 2242 & 9.31 & -0.07458 & $-1.436 \times 10^{-6}$ \\
\hline 29 & 2360 & 0.5 & & \\
\hline
\end{tabular}

Table 3. The deviation of the coordinates of the smoothed contour

\begin{tabular}{|c|c|c|c|c|c|}
\hline$i$ & $x_{i}$ & $y_{i}^{(0)}$ & $y_{i}$ & $\Delta y_{a b s}$ & $\Delta y_{o}$ \\
\hline 11 & 295 & 85.59 & 85.59 & 0 & $0.00 \%$ \\
\hline 12 & 354 & 91.14 & 91.14 & 0 & $0.00 \%$ \\
\hline 13 & 472 & 99.56 & 99.56 & 0 & $0.00 \%$ \\
\hline 14 & 590 & 104.53 & 104.53 & 0 & $0.00 \%$ \\
\hline 15 & 708 & 105.2 & 105.2 & 0 & $0.00 \%$ \\
\hline 16 & 826 & 105.08 & 105.08 & 0 & $0.00 \%$ \\
\hline 17 & 944 & 101.71 & 101.71 & 0 & $0.00 \%$ \\
\hline 18 & 1062 & 96.78 & 96.31 & -0.47 & $-0.49 \%$ \\
\hline 19 & 1180 & 88.98 & 87.7 & -1.28 & $-1.44 \%$ \\
\hline 20 & 1298 & 80.71 & 79.07 & -1.64 & $-2.03 \%$ \\
\hline 21 & 1416 & 71.98 & 70.42 & -1.56 & $-2.17 \%$ \\
\hline 22 & 1534 & 63.12 & 61.75 & -1.37 & $-2.17 \%$ \\
\hline 23 & 1652 & 54.2 & 53.06 & -1.14 & $-2.10 \%$ \\
\hline 24 & 1770 & 45.21 & 44.35 & -0.86 & $-1.90 \%$ \\
\hline 25 & 1888 & 36.19 & 35.62 & -0.57 & $-1.58 \%$ \\
\hline 26 & 2006 & 27.13 & 26.87 & -0.26 & $-0.96 \%$ \\
\hline 27 & 2124 & 18.08 & 18.1 & 0.02 & $0.11 \%$ \\
\hline 28 & 2242 & 9.04 & 9.31 & 0.27 & $2.99 \%$ \\
\hline 29 & 2360 & 0.5 & 0.5 & 0 & $0.00 \%$ \\
\hline
\end{tabular}




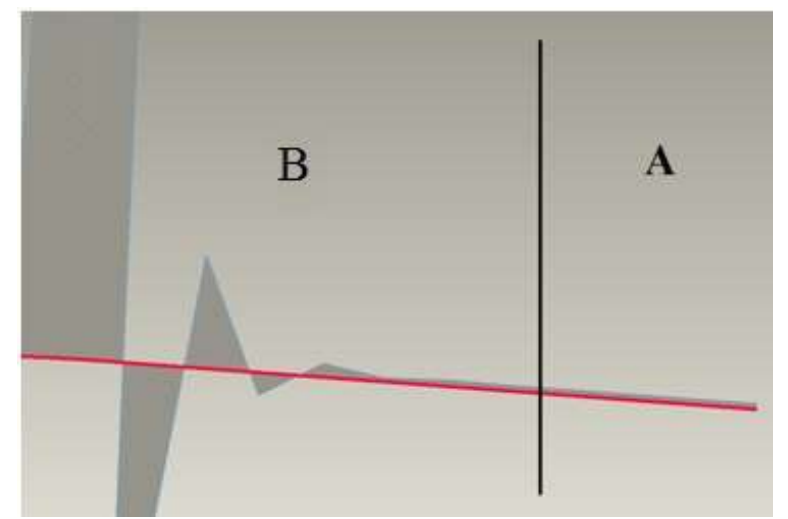

Fig. 7. Graph of the curvature of section "A" smoothed by the developed algorithm.

\section{Conclusion}

The application of the developed algorithm allowed eliminating the existing ad hoc concavity of the profile. Thus, the conducted studies confirm the validity of the proposed criteria for the smoothness of the aerodynamic profile, as well as the effectiveness of the application of the developed algorithm for smoothing profiles that have sections with an extended unregulated concavity, subject to the specified limits on deviation from the original coordinates.

\section{References}

1. Egorov E.V., Erokhin A.P. Smoothing a section of an aerodynamic contour with an unregulated concavity. Flying. 2014. №10. Pp. 54-60.

2. Pocebneva I., Belousov V., Fateeva I., Lukinov V., Folomeeva T. MATEC Web of Conferences Volume 170, 2018. DOI: 10.1051 / matecconf / 201817001120

3. Bityukov Y.I., Deniskin Y.I., Deniskina G.Y. Journal of Physics: Conference Series Volume 944, Issue 1, 2018, DOI:10.1088/1742-6596/944/1/012018

4. I. Pocebneva, V. Belousov, I. Fateeva, V. Lukinov, T. Folomeeva, MATEC Web of Conferences 170, 01120 (2017) DOI: 10.1051 / matecconf / 201817001120

5. Y.I. Bityukov, Y.I. Deniskin, G.Y. Deniskina, Journal of Physics: Conference Series 944-1, 012018 (2018) DOI: 10.1088 / 1742-6596 / 944/1/012018

6. B.V. Boitsov, I.M. Artamonov, Yu.I. Deniskin, Proceedings of the MAI 49, 52 (2011)

7. A.P. Erokhin, Yu.I. Deniskin, Natural and technical sciences 4(66), 270-273 (2013)

8. I.V. Pocebneva, A.V. Ivanova, K.D. Ivarlak, O.V. Ermakova, 8th All-Russian Scientific and Technical Conference with international participation: Modern innovations in science and technology 1, 162-166 (2018)

9. I.M. Artamonov, Yu.I. Deniskin, A.R. Deniskina, Quality and life 4(16), 13-17 (2017)

10. A.P. Erokhin, Yu.I. Deniskin, Internet magazine: Naukovedenie 4(17), 32 (2013) 